\title{
PV-VR: A Virtual Reality Training Application Using Guided Virtual Tours of the Photovoltaic Applied Research and Testing (PART) Lab
}

\section{Dr. Kenneth A. Ritter III, University of Louisiana, Lafayette}

Kenneth Ritter is a concentrating solar power research scientist at the University of Louisiana at Lafayette. Kenneth directed the development of the Virtual Solar Energy Center (VSEC) virtual reality lab at the University of Louisiana at Lafayette. His research interests include solar power, virtual reality, immersive education, and engineering education.

\section{Dr. Terrence L. Chambers P.E., University of Louisiana, Lafayette}

Terrence Chambers currently serves as Director of the Center for Energy Efficiency and Sustainable Energy at the University of Louisiana, Lafayette. His research interests include alternative energy,virtual reality, engineering design and optimization, and artificial intelligence. He is an active member of ASEE and LES, and is a registered Professional Engineer in Louisiana. 


\title{
PV-VR: A virtual reality training application using guided virtual tours of the Photovoltaic Applied Research and Testing (PART) Lab.
}

\begin{abstract}
Leaving the classroom and engaging in learning through first-hand experience has a long tradition in science and engineering education. A virtual field-trip is a way of providing users with some knowledge and virtual experience of a facility without requiring them to physically visit the location. Virtual labs can provide remote-access to various disciplines of Science, Technology, and Engineering (STE) disciplines and are a cost-efficient way for schools and universities to organize high-quality laboratory work. Due to constrictions on time and geographical distances, virtual-labs can be used to share costly equipment and resources, which are otherwise available to a limited number of users. The Photovoltaic (PV) Applied Research and Testing (PART) Lab encompasses a 1.1 MW PV power plant with three solar panel technologies, metrological and radiometer stations, and PV testing lab. PV-VR is an application that uses a scale model of the PART lab for educational interactive lab tours in virtual reality (VR). The actual PV solar power plant is virtualized and virtual representations of all the objects in the plant are inserted into the application. PV-VR takes users on a guided educational tour that allows them to interact with the various technologies to facilitate virtual hands-on learning. The virtual tour is guided by a teacher avatar that explains each technology and initiates animations and interactive educational games with constructive feedback to reinforce learning. PV-VR allows users to learn about solar power technologies and, how power is produced, converted and transmitted. This paper is a summary of the PV-VR application and initial qualitative results from a pilot study.

Introduction

Virtual reality (VR) training provides a portable solution for training and refinement of skills that reduces costs of bringing in specialized educators and travel time as well as risk to the student. Integrating workplace competencies into an application with strategies to support student retention can potentially lead to improved technical STEM education. Twenty-one experimental studies involving VR training since 2013 were analyzed and found HMDs are useful for skills acquisition such as: cognitive skills related to remembering and understanding spatial and visual information and knowledge; psychomotor skills related to head-movement, such as visual scanning or observational skills; and affective skills related to controlling your emotional response to stressful or difficult situations [1]. As the decay of a skill depends greatly on the degree to which the skill was learned, the higher the acquisition environment (e.g., immersive training), the longer the retention [2]. This approach can increase the skilled workforce while decreasing training costs and safety concerns. As the technologies in industry are rapidly changing, providing suitable training programs are of utmost importance. Computer-based learning and other traditional training programs are not adequate in training for various situations that decision makers must deal with [3]. On-the-job training is not practical with high cost and safety concerns in many on-site work situations which have a high focus on productivity. A meta-analysis of nearly 70 educational VR applications gives evidence that VR-based training is
\end{abstract}


an effective means of enhancing learning outcomes [4]. VR training applications can give future engineers the convenience of experiencing complex processes visually by viewing animations in an immersive environment [5]. There has been a consistent increase in publications in VR training in the last 20 years as shown in Figure 1.

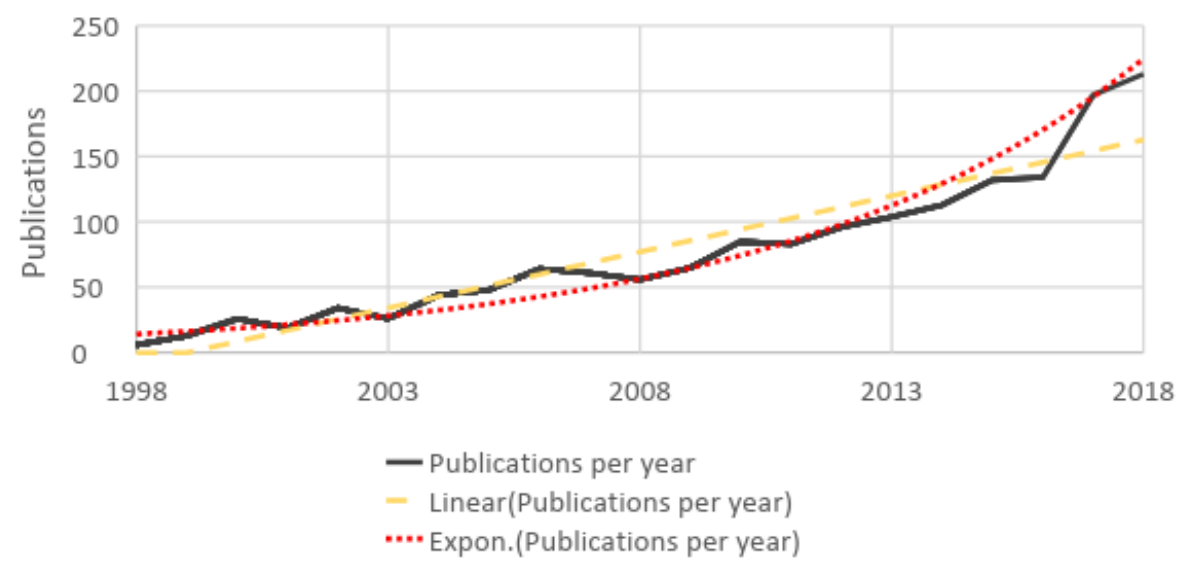

Figure 1: Number of articles per year with titles that contains Virtual Reality and on topic of 'Training' in Web of Knowledge from 1998 to 2018. The linear trend shows the constant growth, while the exponential trend estimated the expected growth.

Publishing articles on VR that involve training has climbed from six in 1998 to 213 in 2018 and will reach over 300 in 2020 following this trend. VR training systems are currently used in many different fields such as: manufacturing, medicine, education, military, as well as training programs for skills of professionals, employees, specialists and managers in prototyping, assembly, drive, flight, surgery, etc. [6].

The use of VR training applications such as virtual labs is a growing research area for professionals and operators in many different fields. Laboratory exercises are required in many fields to provide effective skill acquisition and hands-on experience. Access to these labs for training are not always feasible for many trainees, replicating the lab as a fully software-based virtual lab could potentially solve this issue. In a review of virtual labs, several advantages were identified including cost savings, flexibility in experiments, multiple access, and damage resistance. Also, visual advantages such as transparent equipment covers that reveal motors, gearboxes, and other components were noted (Potkonjak et al., 2016). One study compared actual field trips with immersive virtual field trips (iVFTs) and identified many advantages including cost, time, weather issues, physical inaccessible or dangerous places, online teaching environments, and scale as many field trips are spatially constrained [7]. Many practical problems in education and training can be addressed in VR training applications. The aim of this paper is to provide summary of the PV-VR application design and initial qualitative results from a pilot study.

Method

The PV-VR application was designed initially to give virtual tours of the PART lab while explaining the various solar technologies. This application was developed using the game engine, Unity [8], the digital audio editor, Audacity [9], and the 3D computer graphics applications, 
Solidworks [10], Blender [11], and Maya [12]. Unity is the game engine that provided the platform for adding models, scripts, animations, and building the application. The Unity plugin VRTK (VR Tool Kit) [13], provided grabbing interactions and controls. Blender and Maya were used for 3D modeling of objects in the scene and applying textures. Audacity was used for recording and editing the narration and sound effects. All of the programming was done using C\# in Visual Studio within the Unity game engine. The teacher avatar animations were created with the Cinema Mocap 2 plugin for Unity [14] using the Microsoft Kinect V2 [15].

The test group consisted of forty-four $8^{\text {th }}$ graders, 18 males and 26 females. Students would be given a headset and remote with no instructions and asked to complete the application. Two VR stations were used at the middle school shown in Figure 2.

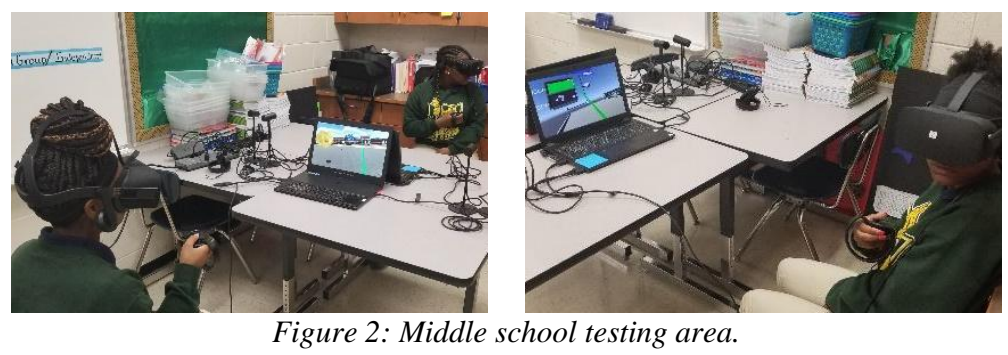

The application took between 5 and 10 minutes to complete depending on how much the user looked around the environment without continuing to the next lesson or area. Laptops were used with the Oculus CR1 for display and Oculus Touch remote for interaction. Following the application students were given feedback surveys on the positive and negative aspects of the application and suggestions for improvement.

\section{PV-VR Application Content}

The PV-VR consists of several interactive educational areas intended to give students a brief overview of the solar resource, photovoltaics, DC to AC inverters, and various PV technologies at the PART lab. The actual PART lab shown in Figure 3 is sited near the university and tests several solar technologies side-by-side. The PART lab is modular allowing testing of multiple different solar technologies, including polycrystalline, monocrystalline, and thin-film, simultaneously.

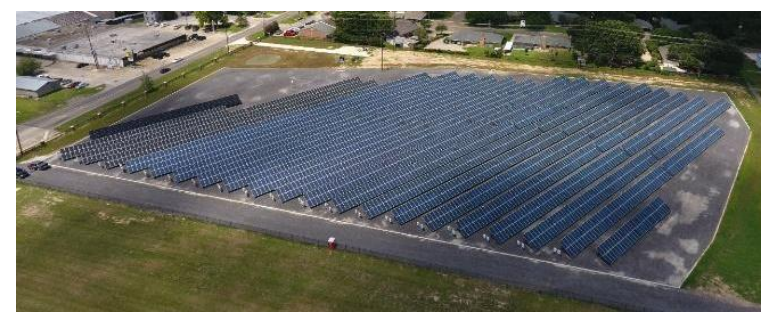

Figure 3: PART Lab overhead view. 
Initially a scale model of the PART lab is modeled in SolidWorks. This model was imported into Maya to add photorealistic textures to all of the objects. The model is then optimized to increase rendering speed so that the frames per second (FPS) is high enough for a comfortable VR experience. With Microsoft Kinect V2, the motions and voice of the solar energy expert are captured and applied to the rigged virtual instructor avatar. The Virtual Interactive Solar Instructor Robot, nicknamed VISIR, served as the guide throughout the application and was able to provide visual and vocal interaction with students during gameplay. The scenes of the application include a headset check, welcome introduction, solar resource, PV power, inverter, and finally the three PV technologies thin-film, monocrystalline, and polycrystalline.

The design of an efficient VR training environment includes a combination of epistemology, pedagogy, methodology and instructional strategy to impact the learner's perception, cognition and actions [16]. The application was designed to be used without external instruction allowing the student to stay immersed until completed. Initially there is a headset check where students are asked by VISIR if the visual and audio are ok and if the headset is comfortable. The controls are explained with a diagram, although one button controls are used to minimize difficulty. After confirming the headset is working properly, students are given a brief introduction of the PV-VR application and the actual PART lab. The solar resource that the earth has was then explained with accompanying animation shown in Figure 4.

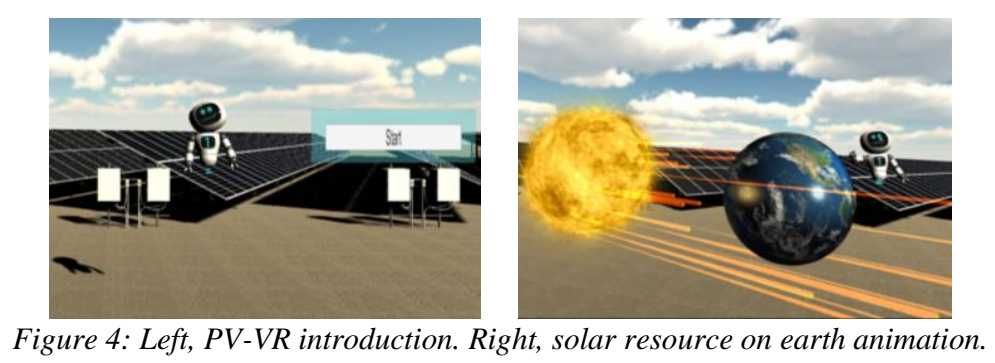

Following the solar resource animation, fundamentals on solar power generation were explained to students covering solar thermal power and photovoltaics. In the PV power area, an animation was given on photons dislodging electrons to produce direct current shown in Figure 5, left.

Students are then directed to participate in a photon shooter activity where photos are shot at the negative doped silicon to free electrons where direct current powers a light bulb shown in Figure 5 , right.
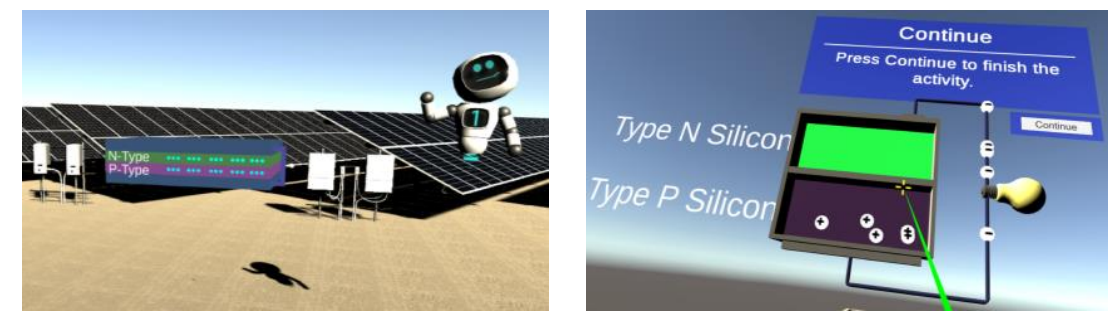

Figure 5: Left, direct current from photons animation. Right, photon shooter activity to power the lightbulb.

Students are given an explanation that most appliances run on alternating current (AC) but solar cells generate direct current (DC). Therefore, DC runs from the solar cells through an inverter to 
convert to AC current. An animation is shown, Figure 6, where DC current travels from the solar panels through the inverter creating AC current to supply power to the home.

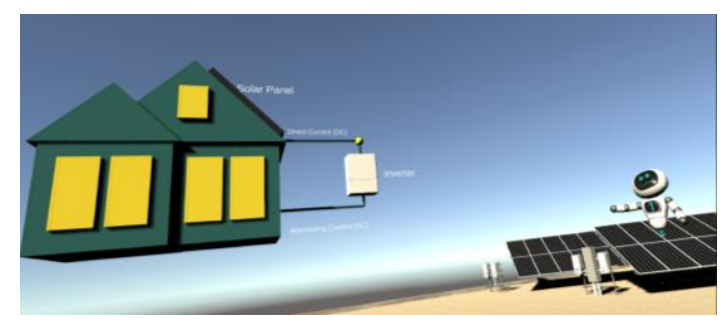

Figure 6: Inverter animation.

The three different PV technologies at the PART Lab, thin-film, monocrystalline, and polycrystalline are explained in the final area shown in Figure 7.
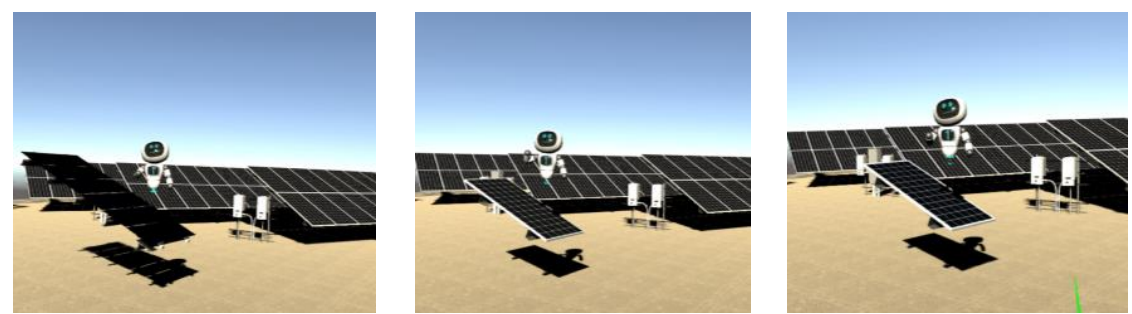

Figure 7: The three different PV technologies at the PART Lab, from left to right, thin-film, monocrystalline, and polycrystalline.

A closeup model was presented while VISIR explains how each panel was produced along with the advantages and disadvantages of each one in terms of efficiency and cost.

Results

The application consisted of audible instruction, informative animations, and required game-like interaction to reinforce learning. The instructions were given in a way to minimize assistance needed with completing the application, and only 3 students out of the 44 tested needed assistance. Once students completed the application they were given feedback surveys on the positive and negative aspects and suggestions for improving. As shown in Table 1, 84\% of students gave positive comments on the VR experience and nearly $50 \%$ commented that it was fun.

Table 1: Positive comments following application.

\begin{tabular}{|l|c|c|c|}
\hline Positive Comments & \% Male & \% Female & \% Total \\
\hline Like VR & $67 \%$ & $96 \%$ & $84 \%$ \\
\hline Fun & $44 \%$ & $50 \%$ & $48 \%$ \\
\hline Informative & $28 \%$ & $42 \%$ & $36 \%$ \\
\hline Immersiveness & $0 \%$ & $35 \%$ & $20 \%$ \\
\hline Gameplay & $17 \%$ & $31 \%$ & $25 \%$ \\
\hline Like robot teacher & $11 \%$ & $19 \%$ & $16 \%$ \\
\hline Graphics & $17 \%$ & $12 \%$ & $14 \%$ \\
\hline
\end{tabular}


Of the 44 students completing the application $29 \%$ more females than males made positive comments about liking the VR experience. $35 \%$ of females commented something positive regarding the immersiveness of the application whereas no males commented on this. $15 \%$ more females commented on the application being informative and 14\% more gave positive remarks on the gameplay or interaction. Students were also asked to give negative feedback and the results are presented in Table 2.

Table 2: Negative comments following application.

\begin{tabular}{|l|c|c|c|}
\hline Negative Comments & \% Male & \% Female & \% Total \\
\hline Blurry & $17 \%$ & $19 \%$ & $18 \%$ \\
\hline Audio/Volume & $11 \%$ & $12 \%$ & $11 \%$ \\
\hline Confusing & $6 \%$ & $8 \%$ & $7 \%$ \\
\hline Add more surroundings & $17 \%$ & $0 \%$ & $7 \%$ \\
\hline To short & $0 \%$ & $12 \%$ & $7 \%$ \\
\hline Headset heavy & $0 \%$ & $12 \%$ & $7 \%$ \\
\hline Needs improvement & $6 \%$ & $4 \%$ & $5 \%$ \\
\hline Scared to break & $6 \%$ & $4 \%$ & $5 \%$ \\
\hline
\end{tabular}

The most common negative feedback was that the application was blurry. Following this several students commented that the audio was too low, distracting background noises were heard or that there was an echo. $7 \%$ of all students mentioned that the application was confusing. Several males mentioned that there should be more surrounding objects and several females mentioned that the application was too short and that the headset was heavy. Only a few students had prior experience with VR systems and two students commented that they have VR systems at their house.

Discussion and Conclusion

The overwhelming majority of students gave positive remarks regarding the VR experience, with nearly $50 \%$ stating that it was fun and over a third stating that it was informative. Students were excited and were primed for accepting VR training as a part of their educational curriculum. Many users commented the display was blurry after the experiment. If the headset is not sitting right on the users face, it appears blurry. In the application, the avatar asks users to adjust the headset, however, many of the students did not touch the headset. If the headset wasn't lined up properly with their eyes, they experienced a blurry display. A few students commented that they were scared to break the headset and possibly feared adjusting might cause it to break. This initial study obtained valuable feedback and suggestions to further development of the PV-VR training application.

Future Study Design

The future goal of this study aims at evaluating the knowledge retention after immersive and non-immersive skill training methods. Actual field trip and virtual field trips can also be compared quantitatively and qualitatively. To increase student engagement, a system of rewards 
and goals to motivate students as well as feedback will be given with interactive cues that prompt learning.

Future updates with the PV-VR application will incorporate the radiometer and weather station, transformers and Vista switch station, and PV testing equipment at the PART lab including LED custom I-V flash test system, Atonometrics light soaking system, Therosensorik thermal imaging system, 32 channel daystar multi-tracer 5, and custom angle of incidence tilt table.

\section{References}

[1] L. Jensen and F. Konradsen, "A review of the use of virtual reality head-mounted displays in education and training," Educ. Inf. Technol., pp. 1-15, 2017.

[2] G. R. Loftus, “Observations: Evaluating forgetting curves,” J. Exp. Psychol. Learn. Mem. Cogn., vol. 11, no. 2, pp. 397-406, 1985.

[3] P. Wang, P. Wu, J. Wang, H.-L. Chi, and X. Wang, "A Critical Review of the Use of Virtual Reality in Construction Engineering Education and Training," Int. J. Environ. Res. Public Health, vol. 15, no. 6, p. 1204, 2018.

[4] Z. Merchant, E. T. Goetz, L. Cifuentes, W. Keeney-Kennicutt, and T. J. Davis, "Effectiveness of Virtual Reality-Based Instruction on Students' Learning Outcomes in K12 and Higher Education: A Meta-analysis," Comput. Educ., vol. 70, pp. 29-40, 2014.

[5] K. A. Ritter III, C. W. Borst, and T. L. Chambers, "Virtual Solar Energy Center Case Studies," Comput. Educ. J., vol. 9, no. 3, 2018.

[6] S. Borsci, G. Lawson, and S. Broome, "Empirical evidence, evaluation criteria and challenges for the effectiveness of virtual and mixed reality tools for training operators of car service maintenance," Comput. Ind., vol. 67, pp. 17-26, 2015.

[7] A. Klippel et al., "Transforming Earth Science Education Through Immersive Experiences - Delivering on a Long Held Promise," Br. J. Educ. Technol., pp. 1-14, 2018.

[8] “Unity 3D.” [Online]. Available: https://unity3d.com/. [Accessed: 13-Feb-2019].

[9] "Audacity." [Online]. Available: https://www.audacityteam.org/. [Accessed: 13-Feb2019].

[10] Dassault Systèmes SolidWorks Corporation, "Solidworks, 3D CAD Design Software," 2018. [Online]. Available: https://www.solidworks.com/. [Accessed: 26-Apr-2019].

[11] “Blender." [Online]. Available: https://www.blender.org/. [Accessed: 13-Feb-2019].

[12] Autodesk, "Maya." [Online]. Available: https://www.autodesk.com/products/maya/overview. [Accessed: 13-Feb-2019].

[13] “VRTK - Virtual Reality Toolkit." [Online]. Available: https://vrtoolkit.readme.io/docs. [Accessed: 13-Feb-2019].

[14] Cinema Suite, "Cinema Mocap 2 - Markerless Motion Capture," 2018. [Online]. 
Available: $\quad$ https://assetstore.unity.com/packages/tools/animation/cinema-mocap-2markerless-motion-capture-56576. [Accessed: 26-Apr-2019].

[15] Microsoft, "Microsoft Kinect - Windows app development," 2018. [Online]. Available: https://developer.microsoft.com/en-us/windows/kinect. [Accessed: 26-Apr-2019].

[16] S. K Babu, S. Krishna, U. R, and R. R. Bhavani, "Virtual Reality Learning Environments for Vocational Education: A Comparison Study with Conventional Instructional Media on Knowledge Retention.," 2018 IEEE 18th Int. Conf. Adv. Learn. Technol., pp. 385-389, 2018. 